\title{
Protective role of magnesium isoglycyrrhizinate in non-alcoholic fatty liver disease and the associated molecular mechanisms
}

\author{
QIAN XU $^{1 *}$, JI WANG $^{1 *}$, FEIFEI CHEN $^{1}$, KAISU LIN $^{1}$, MINGAO ZHU $^{1}$, LEI CHEN $^{1}$, \\ XIUMIN ZHOU ${ }^{2}$, CHONG $\mathrm{LI}^{3}$ and HONG ZHU ${ }^{1}$ \\ ${ }^{1}$ Department of Oncology, The Second Affiliated Hospital of Soochow University, Suzhou, Jiangsu 215004; \\ ${ }^{2}$ Department of Oncology, The First Affiliated Hospital of Soochow University, Suzhou, Jiangsu 215006; \\ ${ }^{3}$ CAS Key Laboratory of Infection and Immunity, Institute of Biophysics, \\ Chinese Academy of Sciences (CAS), Beijing 100101, P.R. China
}

Received November 13, 2015; Accepted May 13, 2016

DOI: $10.3892 /$ ijmm.2016.2603

\begin{abstract}
Non-alcoholic fatty liver disease (NAFLD) is one of the most common liver diseases worldwide and there is an urgent need to identify effective pharmacological strategies to treat NAFLD. For this purpose, in the present study, we examined the the possible molecular mechanisms responsible for the effects of $\mathrm{MgIG}$ and the protective effects of $\mathrm{MgIG}$ in a model of NAFLD. The human hepatic L02 cell line and oleic acid were employed to establish an in vitro model of NAFLD. The CCK-8 assay, Hoechst 33258 staining and Annexin V-PI staining were performed in order to evaluate cell viability and apoptosis. Oil red $\mathrm{O}$ staining was used to detect lipid accumulation within the L02 cells. We found that MgIG significantly inhibited lipid accumulation and protected the L02 cells against lipid accumulation-induced apoptosis. Key molecules involved in unfolded protein response (UPR) signaling were upregulated in lipid-overloaded hepatic cells whereas MgIG suppressed the activation of the UPR. Furthermore, MgIG significantly inhibited the expression of the downstream inflammatory cytokines which had been induced by lipid accumulation. Taken together, these findings suggest that the activation of UPR signaling induces the
\end{abstract}

Correspondence to: Professor Chong Li, CAS Key Laboratory of Infection and Immunity, Institute of Biophysics, Chinese Academy of Sciences (CAS), 15 Datun Road, Beijing 100101, P.R. China E-mail: lichong87@126.com

Professor Hong Zhu, Department of Oncology, The Second Affiliated Hospital of Soochow University, 1055 Sanxiang Road, Suzhou, Jiangsu 215004, P.R. China

E-mail: zhuhong_jasmine@suda.edu.cn

*Co-first authorship

Key words: non-alcoholic fatty liver disease, magnesium isoglycyrrhizinate, unfolded protein response, nuclear factor- $\kappa \mathrm{B}$, inflammatory cytokines expression of inflammatory cytokines through the activation of nuclear factor- $\kappa \mathrm{B}(\mathrm{NF}-\kappa \mathrm{B})$ in lipid-overloaded hepatic cells. In addition, MgIG may suppress the activation of UPR signaling thereby protecting hepatic cells from NAFLD-induced injury.

\section{Introduction}

Non-alcoholic fatty liver disease (NAFLD) is characterized by the accumulation of fat in the livers of individuals who are abstinent from alcohol or drink infrequently and do not have other liver diseases such as hepatitis B and C, alcoholic disease, metabolic liver disease and autoimmune diseases. The histological pattern of NAFLD may progress from non-alcoholic fatty liver into non-alcoholic steatohepatitis (NASH), liver fibrosis, cirrhosis and even hepatocellular carcinoma (HCC) (1). It is now one of the most common liver diseases worldwide. Approximately 10 to $40 \%$ of adults in the USA and $20 \%$ in other developed countries suffer from NAFLD (2).

The pathogenesis and underlying mechanisms of NAFLD remain to be elucidated. Accumulating evidence has verified that NAFLD is associated with obesity, type 2 diabetes, hyperlipidemia and other comorbid conditions. The most widely supported theory proposes that insulin resistance is the key mechanism responsible for the metabolic abnormalities and may cause NAFLD. Current treatment relies on lifestyle modifications including long-term weight management and regular physical exercise. Studies have been performed for the purpose of developing novel treatments for NAFLD including insulin-sensitizers, antioxidants, lipid-lowering drugs, pentoxifylline and cannabinoid receptor antagonists. However, the current pharmacological treatment options for the disease remain unsatisfactory (3-6).

The endoplasmic reticulum (ER) is an organelle in cells which is important for the folding of protein molecules and the transport of synthesized proteins. Failure of the ER's adaptive capacity results in the activation of the unfolded protein response (UPR), which intersects with many different inflammatory and stress signaling pathways. Increasing evidence has demonstrated that the UPR is activated in NAFLD and may play an important role in the development and progression of 
this disease (7-9). 4-Phenylbutyric acid (4-PBA), a chemical chaperone, is used to suppress the activation of the UPR (10).

Previous studies have indicated that lipid accumulation in the liver may regulate the hepatic production of proinflammatory cytokines, including interleukin (IL)-6, transforming growth factor (TGF)- $\beta$ and basic fibroblast growth factor (bFGF) through nuclear factor- $\kappa \mathrm{B}(\mathrm{NF}-\mathrm{kB})$ activation and downstream cytokine production (11-13).

Magnesium isoglycyrrhizinate (MgIG), a traditional herbal remedy extracted from the roots of the plant Glycyrrhiza glabra, is a magnesium salt of $18 \alpha$-glycyrrhizic acid stereoisomer. Increasing evidence indicates that $\mathrm{MgIG}$ acts as an anti-inflammatory and hepatoprotective agent as it protects hepatic cells against tissue injury, improves liver function and suppresses inflammatory responses in the liver (14-16). A previous study demonstrated that MgIG maintained cell viability and markedly decreased oleic acid (OA)-induced cell apoptosis and lipid accumulation (17). It has also been demonstrated that MgIG provided protection against various organ injuries and diseases, including alcoholic liver disease and lung injury induced by paraquat poisoning as a clinical medication (18-20). However, the effect of MgIG in the treatment of NAFLD, particularly with regard to the effect of MgIG on lipid-overloaded hepatic cells as well as the underlying molecular mechanisms remain unknown.

The aim of the study was to explore the the possible molecular mechanisms responsible for the effects of MgIG including activation of the UPR, NF- $\mathrm{kB}$ activation and associated inflammatory factor expression in hepatic L02 cells, as well as to examine the protective effects of MgIG in a model of NAFLD.

\section{Materials and methods}

Cell line and $\mathrm{OA} / \mathrm{MgIG}$ treatment. The human hepatic cell line L02 was obtained from the Peking University Health Science Center (Beijing, China). The L02 cells were cultured in RPMI-1640 medium (Biological Industries, Beit-Haemek, Israel), at $37^{\circ} \mathrm{C}$ in a $5 \% \mathrm{CO}_{2}$ humidified atmosphere. All media were supplemented with $10 \%$ fetal bovine serum and $1 \%$ Pen-Strep solution (both from Biological Industries).

The cells were seeded at either $5 \times 10^{5}$ in $2 \mathrm{ml}$ complete medium in a 6 -well plate, or at $1 \times 10^{4}$ in $200 \mu 1$ medium in a 96-well plate. The cells were grown in fresh medium until $50-60 \%$ confluent and treated with medium alone or supplemented with either OA (2 mM) (Sigma, St. Louis, MO, USA) or OA coupled with MgIG (Chia-tai Tianqing Pharmaceutical Co. Ltd, Lianyungang, China) or 4-PBA (Sigma) for $24 \mathrm{~h}$. The cells were then cultured for another $24 \mathrm{~h}$ after treatment as described above. Specifically, the cells were cultured with the following treatments: i) RPMI-1640 medium alone as the control group, ii) RPMI-1640 with OA as the OA group, iii) RPMI-1640 with OA and MgIG as the $\mathrm{OA}+\mathrm{MgIG}$ group, and iv) RPMI-1640 with OA and 4-PBA as the OA+PBA group.

Cell Counting Kit-8 (CCK-8) assay. After individual treatments, cell viability was measured with CCK- 8 according to the manufacturer's instructions (Dojindo Molecular Technologies, Inc., Kumamoto, Japan). CCK-8 (10 $\mu \mathrm{l})$ was added to each well and then the cells were incubated for another $3 \mathrm{~h}$ in a incubator at $37^{\circ} \mathrm{C}$ and $5 \% \mathrm{CO}_{2}$. The $\mathrm{OD}$ values of the cells from the five different groups were measured at $450 \mathrm{~nm}$ using an ELISA reader (Bio-Tek Instruments, Inc., Winooski, VT, USA). Each assay was performed in triplicate.

Oil red $O$ staining. The $\mathrm{L} 02$ cells were seeded in 6-well plates and cultured in RPMI-1640 medium or supplemented with either $\mathrm{OA}$ and/or MgIG as described above. The culture medium was discarded and the cells were washed with phosphate-buffered saline (PBS) twice and then fixed with 4\% paraformaldehyde for 20 min. Oil red O working solution (Sigma-Aldrich, St. Louis, MO, USA) was added to each well and incubated for $15 \mathrm{~min}$ at room temperature. The cells were then rinsed with $60 \%$ isopropyl alcohol once and then rinsed with PBS twice. Images were then captured under an upright microscope (magnification, x400; BX53; Olympus, Tokyo, Japan).

Hoechst 33258 staining. The effect of MgIG and OA on apoptosis were determined by fluorescence microscopy following staining with the DNA binding fluorophore Hoechst 33258 . Briefly, the L02 cells were seeded in 6-well plates for $24 \mathrm{~h}$ and treated with either growth medium or medium containing OA with or without MgIG. The cells were washed twice with PBS, fixed with $4 \%$ paraformaldehyde for $10 \mathrm{~min}$, and stained with $5 \mathrm{mM}$ Hoechst 33258 (Dojindo Molecular Technologies, Inc.) for $10 \mathrm{~min}$ at room temperature. The cells were washed with PBS twice before images were captured under a fluorescence microscope (Olympus) at $340 \mathrm{~nm}$ excitation (magnification, $\mathrm{x} 400$ ).

Annexin V-FITC/propidium iodide (PI) staining and flow cytometric analysis. Following the $\mathrm{OA}$ and MgIG treatment for $24 \mathrm{~h}$ as described above, apoptosis was detected using the Annexin V-FITC Apoptosis Detection kit (Dojindo Molecular Technologies, Inc.) according to the manufacturer's instructions. Individually treated L02 cells were collected by removing the medium and washed twice with PBS. The cells were gently resuspended in $100 \mu 1$ binding solution. Annexin V-FITC ( $5 \mu \mathrm{l}$ ) was added to the cells, followed by $5 \mu \mathrm{l}$ PI solution. The L02 cells were then incubated at room temperature in the dark for $15 \mathrm{~min}$. Subsequently, $400 \mu \mathrm{l}$ 1X Annexin V binding solution was added. The cells were analyzed by flow cytometry using a BD FACSCalibur flow cytometer (Becton-Dickinson, Franklin Lakes, NJ, USA) within $1 \mathrm{~h}$. The number of apoptotic cells was then calculated using FlowJo software.

Reverse transcription-quantitative polymerase chain reaction $(R T-q P C R)$. Following a 24-h incubation, total RNA was extracted from the L02 cells using TRIzol reagent (Sigma) and reverse transcribed according to the manufacturer's instructions. Briefly, the SuperReal PreMix RT-PCR kit (Tiangen Biotech Co.,Ltd.,Beijing, China) was used to perform Real-Time PCR and the Golden Fast PCR kit (Tiangen Biotech Co., Ltd.) was used to perform PCR. Isolated total RNA $(2 \mu \mathrm{g})$ was reverse transcribed into cDNA using the High Capacity cDNA Reverse Transcription kit (Applied Biosystems, Foster City, CA, USA). The primers used for IL- 6 , TGF- $\beta$, bFGF, NF- $\kappa B$, $\mathrm{BiP}, \mathrm{C} / \mathrm{EBP}$ homologous protein (CHOP), X-box binding protein 1 (XBP-1), activating transcription factor 6 (ATF-6) and $\beta$-actin are presented in Table I. The primers were selected 
Table I. Primer sequences used for RT-qPCR.

\begin{tabular}{|c|c|}
\hline Gene & Primer sequence $\left(5^{\prime} \rightarrow 3^{\prime}\right)$ \\
\hline $\mathrm{BiP}$ & $\begin{array}{l}\text { Forward: CTAATGGTGGAAACCCACAACG } \\
\text { Reverse: TATCGCCAGGAATTGTTGCTG }\end{array}$ \\
\hline $\mathrm{CHOP}$ & $\begin{array}{l}\text { Forward: GCTCAGGAGGAAGAGGAGGA } \\
\text { Reverse: CCTGCTTGAGCCGTTCATT }\end{array}$ \\
\hline XBP-1 & $\begin{array}{l}\text { Forward: CAGACTACGTGCACCTCTGC } \\
\text { Reverse: GGCTGGTAAGGAACTGGGTC }\end{array}$ \\
\hline ATF-6 & $\begin{array}{l}\text { Forward: TCCTCGGTCAGTGGACTCTTA } \\
\text { Reverse: CTTGGGCTGAATTGAAGGTTTTG }\end{array}$ \\
\hline $\mathrm{NF}-\kappa \mathrm{B}$ & $\begin{array}{l}\text { Forward: GAAGCACGAATGACAGAGGC } \\
\text { Reverse: GCTTGGCGGATTAGCTCTTTT }\end{array}$ \\
\hline IL-6 & $\begin{array}{l}\text { Forward: GGCACTGGCAGAAAACAACC } \\
\text { Reverse: TGGCATTTGTGGTTGGGTCA }\end{array}$ \\
\hline TGF- $\beta$ & $\begin{array}{l}\text { Forward: CTAATGGTGGAAACCCACAACG } \\
\text { Reverse: ATATCGCCAGGAATTGTTGCTG }\end{array}$ \\
\hline bFGF & $\begin{array}{l}\text { Forward: AGTGTGTGCTAACCGTTACCT } \\
\text { Reverse: ACTGCCCAGTTCGTTTCAGTG }\end{array}$ \\
\hline$\beta$-actin & $\begin{array}{l}\text { Forward: GTCACCAACTGGGACGACAT } \\
\text { Reverse: AGGGATAGCACAGCCTGGAT }\end{array}$ \\
\hline
\end{tabular}

CHOP, C/EBP homologous protein; XBP-1, X-box binding protein 1; ATF-6, activating transcription factor 6 ; NF- $\mathrm{BB}$, nuclear factor- $\mathrm{\kappa B}$; IL-6, interleukin-6; TGF- $\beta$, transforming growth factor- $\beta$; bFGF, basic fibroblast growth factor.

using the NCBI/Primer-BLAST program (www.ncbi.nlm. nih.gov/tools/primer-blast/) and were synthesized by Sangon Biotech (Shanghai, China). In addition, the PCR products were also separated in a $2 \%$ agarose gel and analyzed using Image Quant software. Quantitative (real-time) PCR was performed on a 7300 Real-Time PCR system (Applied Biosystems). Cycle threshold $(\mathrm{Ct})$ values were normalized to $\beta$-actin and PCR system reactions were run and calculated in triplicates.

Protein extraction and western blot analysis. After individual treatments, the L02 cells were harvested, rinsed with PBS twice and lysed in RIPA buffer containing $10 \mathrm{mM}$ phosphate buffer, $150 \mathrm{mM} \mathrm{NaCl}, 2 \mathrm{mM}$ EDTA, $0.1 \%$ SDS, $1 \%$ sodium deoxycholate, $1 \%$ Triton $\mathrm{X}-100,1 \mathrm{mM}$ sodium orthovanadate and protease inhibitors for $30 \mathrm{~min}$ on ice. Protein concentrations were determined using a BCA Protein Assay kit (Beyotime Institute of Biotechnology, Shanghai, China). The samples were boiled for $10 \mathrm{~min}$ at $100^{\circ} \mathrm{C}$ and insoluble material was removed by centrifugation. Total protein $(50 \mu \mathrm{g})$ was separated on $12 \%$ SDS-PAGE gels and transferred to polyvinylidene difluoride (PVDF) membranes. The membranes were blocked with $7 \%$ fat-free milk in $0.5 \%$ Triton X-100-TBS (TBST) and incubated overnight at $4^{\circ} \mathrm{C}$ in blocking solution containing primary antibodies. The membranes were then washed with TBST 3 times and incubated with secondary HRP-conjugated goat anti-rabbit IgG diluted in TBST with $7 \%$ milk for $2 \mathrm{~h}$ at room temperature. Protein bands were developed using chemiluminescence detection reagents and then visualized on
X-ray films. The films were scanned using a GT-1500 scanner (Epson, Tokyo, Japan). $\beta$-actin (Santa Cruz Biotechnology, Inc., Santa Cruz, CA, USA) was used as an internal control. The rabbit polyclonal antibodies against XBP-1 (SAB3500381), CHOP (SAB4500631) or NF- $\kappa$ B protein (SAB4502610) were purchased from Sigma. The rabbit polyclonal antibodies against BiP (sc-33757) or ATF-6 protein (sc-22799) were purchased from Santa Cruz Biotechnology, Inc.

Enzyme-linked immunosorbent assay (ELISA). The expression of IL- 6 , TGF- $\beta$ and bFGF in the L02 cells was detected by ELISA. After a 24-h treatment period, the supernatants were collected and added into the human ELISA kit (Abcam, Cambridge, MA, USA) according to the manufacturer's instructions. The OD value was measured at $450 \mathrm{~nm}$ using an ELISA reader (Bio-Tek Instruments). The expression values were calculated using a standard curve and ELISA was performed with supernatants with each independent culture run in triplicate.

Statistical analysis. All quantitative data are presented as the means \pm SD. Statistical analysis was performed using the ANOVA and Student's t-test with SPSS 17.0 software. A P-value $<0.05$ was considered to indicate a statistically significant difference.

\section{Results}

$M g I G$ increases the viability of LO2 cells following exposure to $O A$. Lipotoxicity in hepatocytes induced by OA has been implicated in the development of NAFLD. OA and hepatic L02 cells have been previously used to establish a model of NAFLD in vitro (21).

In the present study, we first treated the L02 cells with OA and different doses of $\mathrm{MgIG}(0.2,1$ and $5 \mathrm{mg} / \mathrm{ml})$ for $24 \mathrm{~h}$. Compared with the untreated control group, OA significantly reduced the viability of the L02 cells (Fig. 1A). Furthermore, MgIG increased cell viability. The optimal concentration of $\mathrm{MgIG}$ for protecting L02 cell viability was identified as $1 \mathrm{mg} / \mathrm{ml}$ therefore this dose was selected for subsequent experiments (Fig. 1A).

MgIG decreases the accumulation of lipid droplets in L02 cells exposed to $O A$. The Oil red $\mathrm{O}$ staining assay was performed in order to detect the accumulation of intracellular lipid droplets in L02 cells. After incubation with OA, the number of Oil Red O-positive droplets was significantly increased in the L02 cells (Fig. 1B). MgIG treatment significantly reduced the accumulation of Oil Red O-positive droplets in the OA-treated L02 cells (Fig. 1B).

MgIG protects against the OA-induced apoptosis of LO2 cells. We performed Hoechst 33258 staining to evaluate the effect of MgIG on the apoptosis of lipid-overloaded L02 cells. The results indicated that L02 cells exposed to OA for $24 \mathrm{~h}$ exhibited membrane dissolution as well as the formation of debris and nuclear fragmentation, which was indicated by bright blue fluorescence (Fig. 2A). The number of typical apoptotic cells was decreased following treatment with MgIG compared with that in the the cells incubated with OA alone (Fig. 2A). 

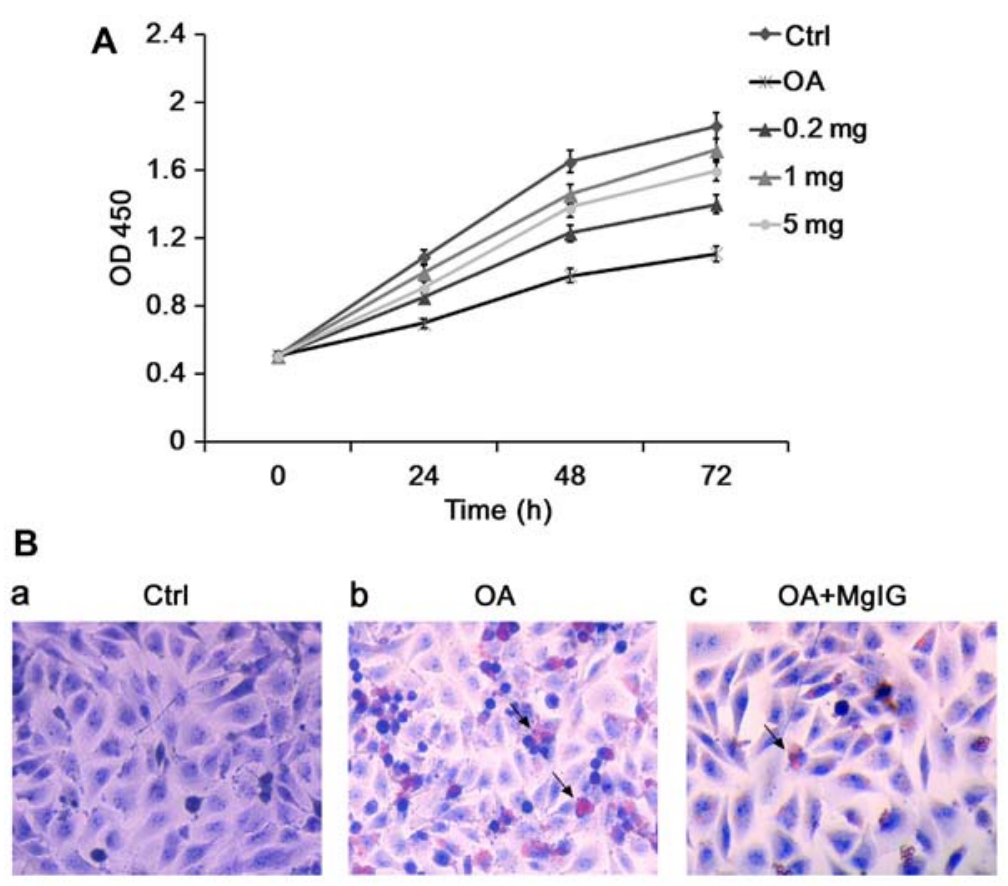

Figure 1. Magnesium isoglycyrrhizinate (MgIG) increases the viability of L02 cells exposed to oleic acid $(\mathrm{OA})$ and decreases the OA-induced accumulation of lipid droplets in L02 cells. (A) Viability of the OA-exposed human hepatic cell line L02 following MgIG treatment. L02 cells were treated with RPMI-1640 alone as the control (Ctrl) or with OA or with OA containing MgIG at concentrations of $0.2,1$, or $5 \mathrm{mg} / \mathrm{ml}$ for $24 \mathrm{~h}$. Cell viability was determined using the Cell Counting Kit-8 (CCK-8) assay as described in the Materials and methods. (B) Oil Red O staining of L02 cells. After a 24-h incubation with different treatments (panel a, control; panel b, OA; and panel c, OA with MgIG) the L02 cells were stained with Oil Red O and observed under an microscope (magnification, x400). Black arrows indicate Oil Red O-stained lipid droplets.

A a

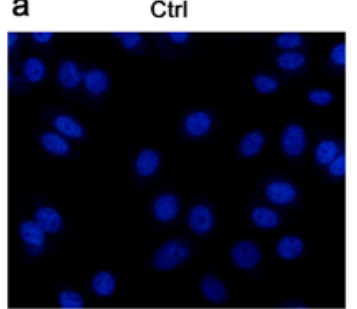

B

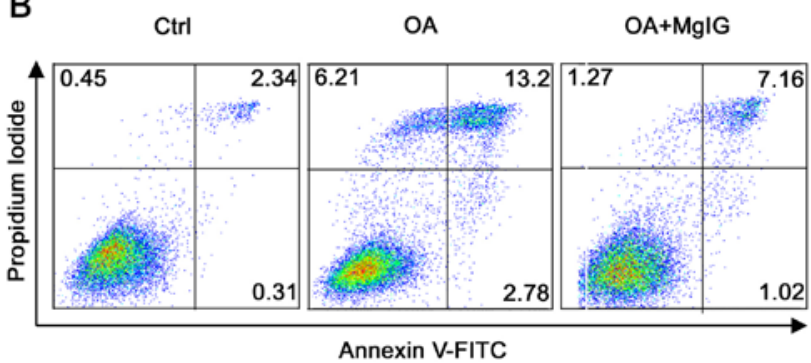

b
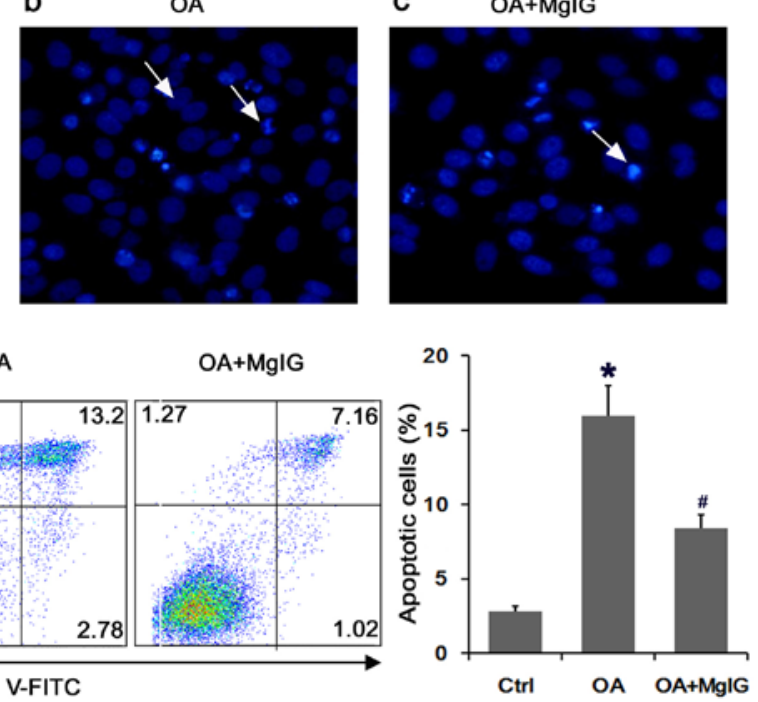

Figure 2. Magnesium isoglycyrrhizinate (MgIG) protects against the oleic acid (OA)-induced apoptosis of L02 cells. (A) Fluorescence images of L02 cells treated individually for $24 \mathrm{~h}$ and stained with Hoechst 33258, then imaged under a fluorescence microscope (panel a, control (Ctrl); panel b, OA; and panel c, OA with MgIG). The staining of apoptotic cells revealed highly condensed, brightly stained nuclei, whereas the normal cells were stained only slightly blue. Apoptotic cells are indicated by white arrows. Magnification, x400. (B) Annexin V-FITC/propidium iodide (PI) double staining. After exposure to OA or OA with MgIG, the cells were harvested and apoptosis was examined by performing flow cytometry after Annexin V/PI staining. The horizontal and vertical axes represent labeling with Annexin V-FITC and PI, respectively. Cells are classified as healthy cells (Annexin $\mathrm{V}^{-}, \mathrm{PI}^{-}$), early apoptotic cells (Annexin $\left.\mathrm{V}^{+}, \mathrm{PI}^{-}\right)$, late apoptotic cells $\left(\right.$ Annexin $\mathrm{V}^{+}, \mathrm{PI}^{+}$), and damaged cells (Annexin $\mathrm{V}^{-}, \mathrm{PI}^{+}$). The bar chart shows the ratio of apoptosis among the different experimental groups. The apoptotic ratio was calculated from the percentage of early apoptotic cells plus the percentage of late apoptotic cells . Data are presented as the means \pm SD of percentage of controls. ${ }^{*} \mathrm{P}<0.05$ vs. control group, ${ }^{~} \mathrm{P}<0.05$ vs. OA group.

The results of Annexin V-PI staining showed that the portion of early- and late-stage apoptotic cells increased markedly following exposure to OA (Fig. 2B). However, MgIG significantly suppressed the percentage of apoptotic cells (Fig. 2B).
MgIG suppresses the lipotoxic effects of OA through UPR signaling activation. $\mathrm{BiP}$ is an ER stress marker that reflects the activation of UPR. The UPR signaling pathway consists of three branches, namely PERK, IRE1 and ATF-6. The activation 

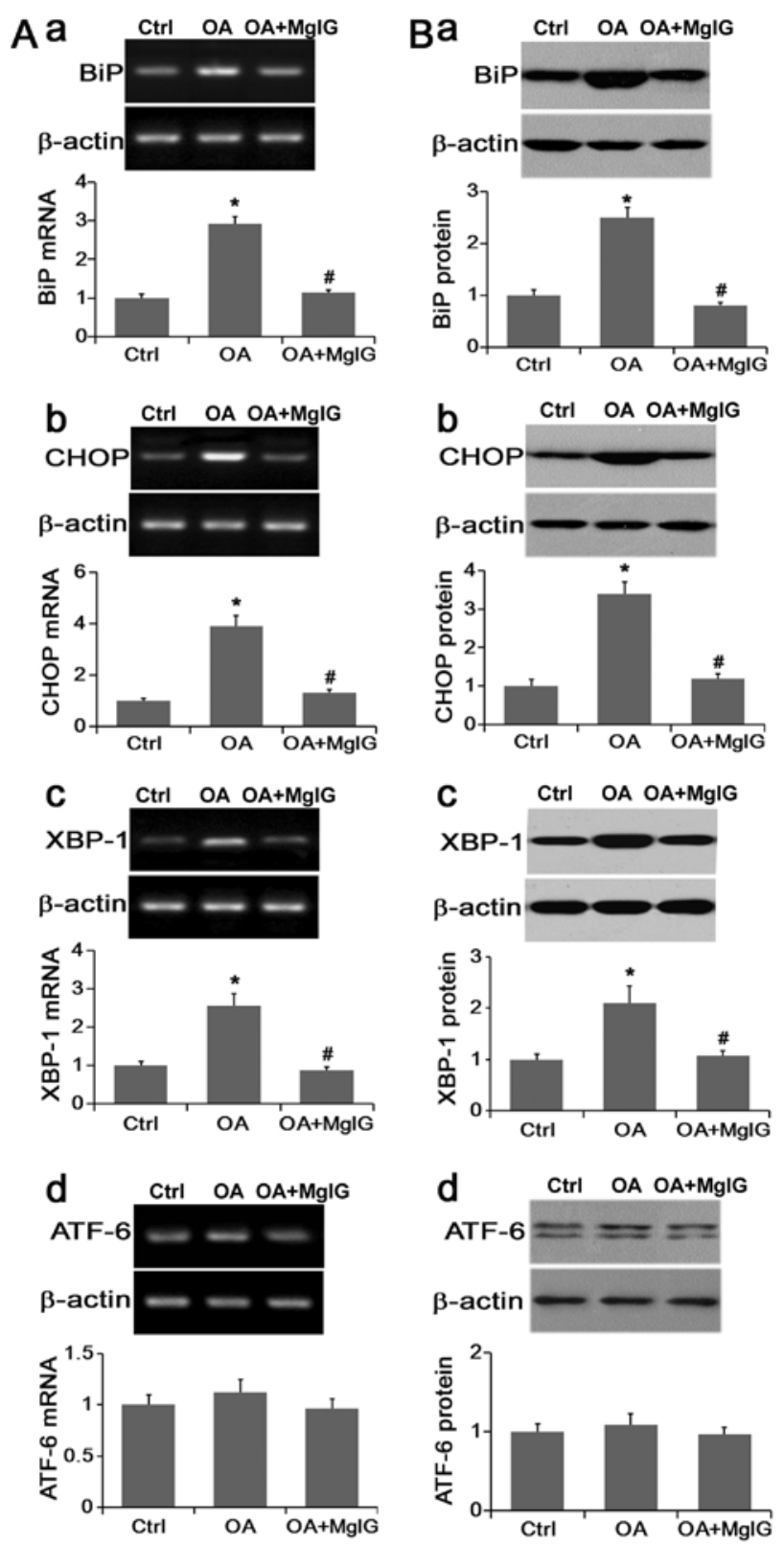

Figure 3. Magnesium isoglycyrrhizinate (MgIG) suppresses the lipotoxic effects of oleic acid (OA) through activation of the unfolded protein response. (A) RT-qPCR analysis of the mRNA expression levels of endoplasmic reticulum stress-related genes following the MgIG treatment and OA exposure of each group. The mRNA expression of (a) BiP, (b) C/EBP homologous protein (CHOP), (c) X-box binding protein 1 (XBP-1) and (d) activating transcription factor 6 (ATF-6) were measured by quantitative (real-time) PCR and normalized to the gene $\beta$-actin. (B) Western blot analysis of the endoplasmic reticulum (ER) stress-related genes following the MgIG treatment and OA exposure of each group. The protein expression of (a) BiP, (b) CHOP, (c) XBP-1 and (d) ATF-6 were normalized to $\beta$-actin. Data are presented as the means \pm SD. ${ }^{*} \mathrm{P}<0.05$ vs. control $(\mathrm{Ctrl})$ group, ${ }^{\text {" }} \mathrm{P}<0.05$ vs. OA group. All the experiments were repeated at least 3 times.

of PERK and IRE1 promoted the activation of CHOP and XBP-1, respectively $(9,10)$. RT-qPCR and western blot analysis were performed to evaluate the mRNA and protein expression of BiP, CHOP, XBP-1 and ATF-6, respectively.

Compared with the control group, the expression of Bip, CHOP and XBP-1 was significantly increased in lipid-overloaded L02 cells, and MgIG effectively reversed
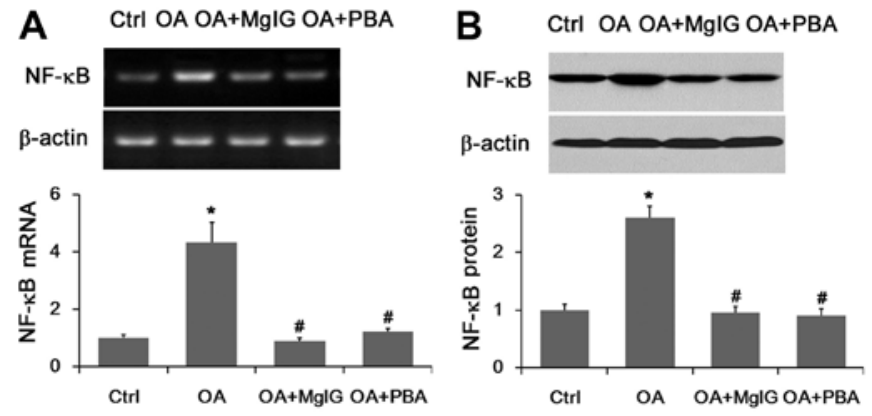

Figure 4. mRNA and protein expression of nuclear factor- $\kappa \mathrm{B}(\mathrm{NF}-\kappa \mathrm{B})$ is altered in lipid-overloaded L02 cells in the presence or absence of magnesium isoglycyrrhizinate (MgIG). (A) RT-qPCR analysis was performed to detect the mRNA expression levels of NF- $\mathrm{kB}$ after MgIG or 4-phenylbutyric acid (4-PBA) treatment with or without oleic acid (OA). $\beta$-actin was used as a loading control. (B) Western blot analysis was performed to evaluate NF- $\kappa \mathrm{B}$ protein expression levels after individual treatments. Expression of NF- $\kappa \mathrm{B}$ protein was normalized to $\beta$-actin. Data are presented as the means $\pm \mathrm{SD}$. ${ }^{*} \mathrm{P}<0.05$ vs. control (Ctrl) group, ${ }^{\#} \mathrm{P}<0.05$ vs. OA group. All the experiments were repeated at least 3 times.

their expression back to normal both at the mRNA and the protein level (Fig. 3A and B). Compared with the other endoplasmic reticulum stress-related genes, ATF-6 expression was not significantly changed by either OA or OA with $\mathrm{MgIG}$ treatment (Fig. 3A and B). These data suggested that OA may induce the activation of UPR signaling mainly through downstream PERK and IRE1 pathways whereas MgIG may greatly suppress OA-induced UPR activation.

$m R N A$ and protein expression of $N F-\kappa B$ is altered in lipid-overloaded LO2 cells in the presence or absence of MgIG. Accumulating evidence indicates that the NF- $\kappa \mathrm{B}$ pathway in the liver is activated and $\mathrm{NF}-\kappa \mathrm{B}$ targets were accordingly elevated by lipid accumulation (11). Our results showed that OA markedly raised the mRNA and protein expression of $\mathrm{NF}-\kappa \mathrm{B}$ (Fig. 4). However, these levels was significantly reduced when the cells were co-treated with MgIG (Fig. 4). The UPR inhibitor 4-PBA decreased the mRNA and protein expression levels of NF- $\kappa \mathrm{B}$ as the positive control (Fig. 4).

Expression of IL-6, TGF- $\beta$ and bFGF is partly regulated in lipid-overloaded LO2 cells in the presence or absence of $M g I G$. The mRNA and protein expression of IL-6, TGF- $\beta$ and bFGF were significantly increased in the lipid-overloaded L02 cells compared with the control groups (Fig. 5A and B). A marked decrease in the mRNA and protein expression of IL-6, TGF- $\beta$ and bFGF was observed following treatment with OA and $\mathrm{MgIG}$ or 4-PBA.

\section{Discussion}

In the present study, we established a model of NAFLD by incubating hepatic L02 cells with OA in vitro. Using this cell model, we examined the role of OA and MgIG in liver cells and the associated molecular signaling events.

A previous study indicated that OA was capable of decreasing cell viability and increasing cell apoptosis markedly (22). It has also been demonstrated that OA may induce the accumulation of lipid droplets in lipid-overloaded 

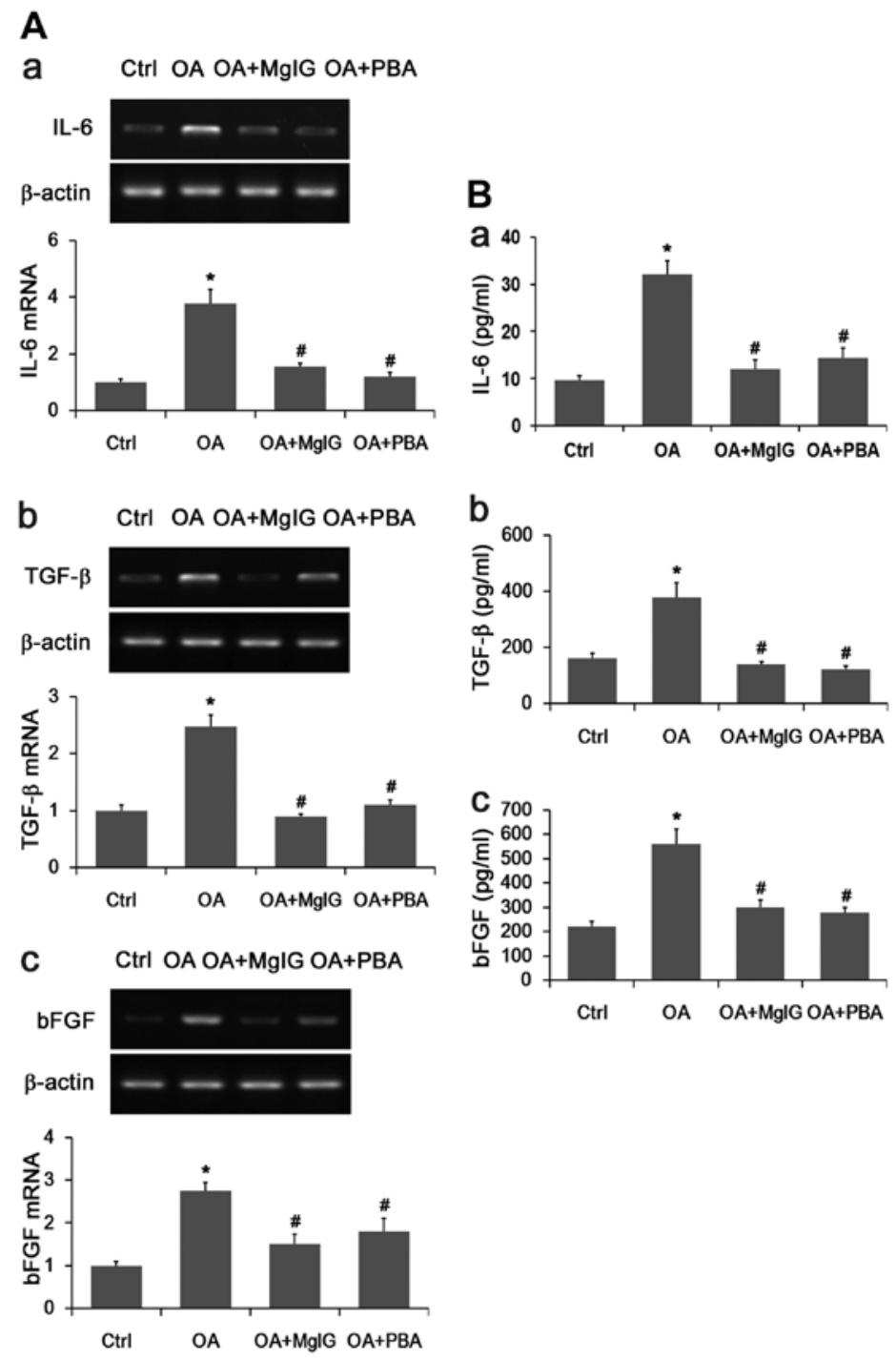

Figure 5. Expression of interleukin (IL)-6, transforming growth factor (TGF)- $\beta$ and basic fibroblast growth factor (bFGF) is partly regulated in oleic acid (OA)-exposed L02 cells in the presence or absence of magnesium isoglycyrrhizinate (MgIG). (A, panels a to c) RT-PCR analysis was performed to evaluate the mRNA expression of IL-6, TGF- $\beta$ and bFGF after OA, MgIG and 4-phenylbutyric acid (4-PBA) treatment. mRNA expression was normalized to $\beta$-actin. (B, panels a to c) Protein expression levels of IL-6, TGF- $\beta$ and bFGF were measured by ELISA. Data are presented as the means \pm SD. ${ }^{*} \mathrm{P}<0.05$ vs. control group, ${ }^{\#} \mathrm{P}<0.05$ vs. OA group. All the experiments were repeated at least 3 times.

L02 cells (23). Our current data are consistent with these findings. In addition, our study suggested that MgIG reduced cell death and decreased the apoptosis rate in lipid-overloaded L02 cells significantly. Moreover, MgIG treatment inhibited OA-induced lipid accumulation in L02 cells.

The UPR is regulated by three ER stress transducer proteins PERK, IRE1, and ATF-6. BiP is an ER stress marker and a central regulator of ER homeostasis as it plays multiple roles in protein folding and the activation of transmembrane ER stress sensors. The accumulation of unfolded proteins sequesters $\mathrm{BiP}$ so it dissociates from transmembrane transducers thereby leading to the activation of these three proteins. Thus, the expression of BiP is thought to be an ER stress marker bound to these three pathways (24). The activation of PERK promotes the activation of CHOP after dissociating from BiP (25). Activated IRE1 $\alpha$ induces the splicing of the mRNA encoding XBP-1 after dissociating from BiP, which is a transcriptional activator that has many targets including ER chaperones and genes involved in ER-associated degradation (ERAD) (26).
After dissociating from BiP, ATF-6 is activated and translocates to the nucleus where it transcriptionally activates ER chaperones and genes associated with ERAD (27).

In the present study, the exposure of L02 cells to OA, markedly upregulated the mRNA and protein expression of BiP, CHOP and XBP-1, which was similar to the findings of a previous study using sodium palmitate (28). However, we were surprised to observe that the mRNA and protein expression of these endoplasmic reticulum stress-related genes were significantly downregulated when treated with MgIG whereas there was no significant change in ATF- 6 levels. The expression of ATF- 6 was upregulated slightly in the lipid-overloaded L02 cells, and suppressed slightly after MgIG treatment. These results suggested that the lipid accumulation caused by OA may activate the UPR and be involved in the activation of the IRE1-XBP-1 and PERK-CHOP pathways. Furthermore, MgIG suppresses the expression of these two pathways.

Previous findings have showed that the activation of NF- $\mathrm{KB}$ pathway may be induced by lipid accumulation (11). A 
close examination of ER stress and UPR pathways has demonstrated many links to major inflammatory and stress signaling networks, including the activation of the $\mathrm{NF}-\kappa \mathrm{B}$ pathways. These findings also indicated that lipid accumulation in the liver leads to the increased hepatic production of inflammatory cytokines, including IL-6, TGF- $\beta$ and bFGF through NF- $\mathrm{B}$ activation and downstream cytokine production (12,29-31).

Changes to inflammatory factors accompany hepatocyte injury and regulate lipid metabolism. IL-6 promotes inflammatory signaling in the liver and increases insulin resistance in hepatocytes (32). However, the underlying mechanism remains unclear. TGF- $\beta$ is a pleiotropic cytokine involved in cell survival, proliferation, differentiation, angiogenesis and wound healing responses; TGF- $\beta$ and bFGF signaling both participate in the fibrogenic response through hepatic stellate cell (HSC) activation and play roles in the progression of fibrosis in advanced NAFLD. TGF- $\beta$ and bFGF signaling in hepatocytes were also involved in insulin resistance (33-35). Insulin resistance represents the most reproducible factor for the development of NAFLD (36).

The present study suggested that OA induces the expression of $\mathrm{NF}-\kappa \mathrm{B}$ and the downstream inflammatory cytokines markedly. 4-PBA, the UPR inhibitor, is used to suppress the activation of the UPR thereby reducing the expression of $\mathrm{NF}-\kappa \mathrm{B}$ and the downstream inflammatory cytokines (10). Our results also clearly showed that the $\mathrm{mRNA}$ and protein expression of NF- $\kappa$ B, IL-6, TGF- $\beta$ and bFGF were significantly downregulated when the lipid-overloaded hepatic cells were treated with $\mathrm{MgIG}$ which was consistent with the effects of 4-PBA.

In conclusion, the data presented in this study demonstrated that IRE1 and PERK signaling branches induced the overexpression of inflammatory cytokines such as IL-6, TGF- $\beta$ and bFGF, through the activation of $N F-\kappa B$ in lipid-overloaded hepatic cells. We suggest that MgIG significantly suppresses the activation of the UPR thereby reducing the downstream inflammatory cytokines and protecting hepatic cells from NAFLD-induced injury.

\section{Acknowledgements}

The present study was supported by grants awarded to H. Zhu from the National Natural Science Foundation of China (no. 81572345) and the Tianqing Liver Disease Research Foundation (no. 20120024).

\section{References}

1. Angulo P and Lindor KD: Non-alcoholic fatty liver disease. J Gastroenterol Hepatol 17. (Suppl): S186-S190, 2002.

2. Başaranoğlu $\mathrm{M}$ and Örmeci N: Nonalcoholic fatty liver disease: diagnosis, pathogenesis, and management. Turk J Gastroenterol 25: 127-132, 2014.

3. Du J, Ma YY, Yu CH and Li YM: Effects of pentoxifylline on nonalcoholic fatty liver disease: a meta-analysis. World $\mathrm{J}$ Gastroenterol 20: 569-577, 2014.

4. Duvnjak M, Lerotić I, Barsić N, Tomasić V, Virović Jukić L and Velagić V: Pathogenesis and management issues for non-alcoholic fatty liver disease. World J Gastroenterol 13 : 4539-4550, 2007.

5. Takahashi Y, Sugimoto K, Inui $\mathrm{H}$ and Fukusato T: Current pharmacological therapies for nonalcoholic fatty liver disease/nonalcoholic steatohepatitis. World J Gastroenterol 21: $3777-3785,2015$
6. Papandreou D and Andreou E: Role of diet on non-alcoholic fatty liver disease: an updated narrative review. World J Hepatol 7: 575-582, 2015

7. Puri P, Mirshahi F, Cheung O, Natarajan R, Maher JW, Kellum JM and Sanyal AJ: Activation and dysregulation of the unfolded protein response in nonalcoholic fatty liver disease. Gastroenterology 134: 568-576, 2008.

8. Zhang K and Kaufman RJ: The unfolded protein response: a stress signaling pathway critical for health and disease. Neurology 66 (Suppl 1): S102-S109, 2006.

9. Kim SR, Kim DI, Kang MR, Lee KS, Park SY, Jeong JS and Lee YC: Endoplasmic reticulum stress influences bronchial asthma pathogenesis by modulating nuclear factor $\kappa \mathrm{B}$ activation. J Allergy Clin Immunol 132: 1397-1408, 2013.

10. Ayala P, Montenegro J, Vivar R, Letelier A, Urroz PA, Copaja M, Pivet D, Humeres C, Troncoso R, Vicencio JM, et al: Attenuation of endoplasmic reticulum stress using the chemical chaperone 4-phenylbutyric acid prevents cardiac fibrosis induced by isoproterenol. Exp Mol Pathol 92: 97-104, 2012.

11. Hotamisligil GS: Endoplasmic reticulum stress and the inflammatory basis of metabolic disease. Cell 140: 900-917, 2010.

12. Cai D, Yuan M, Frantz DF, Melendez PA, Hansen L, Lee J and Shoelson SE: Local and systemic insulin resistance resulting from hepatic activation of IKK-beta and NF-kappaB. Nat Med 11: 183-190, 2005.

13. Li N and Karin M: Signaling pathways leading to nuclear factor-kappa B activation. Methods Enzymol 319: 273-279, 2000.

14. Bao QD, Yang LL and Wang L: Protective effects of magnesium isoglycyrrhizinate against carbon tetrachloride-induced acute liver injury in mice. World Chin J Digestology 16: 1004-1007, 2008.

15. Dong LP, Yu F, Liu J and Mu XM: Protective effect of magnesium isoglycyrrhizinate on acute hepatic injury in mice. China Pharmacy 17: 902-904, 2006.

16. Mao YM, Zeng MD, Chen Y, Chen CW, Fu QC, Cai X, Wu SM, Chen YG, Sun Y, Li J, et al: Magnesium isoglycyrrhizinate in the treatment of chronic liver diseases: a randomized, double-blind, multi-doses, active drug controlled, multi-center study. Zhonghua Gan Zang Bing Za Zhi 17: 847-851, 2009 (In Chinese).

17. Cheng Y, Zhang J, Shang J and Zhang L: Prevention of free fatty acid-induced hepatic lipotoxicity in HepG2 cells by magnesium isoglycyrrhizinate in vitro. Pharmacology 84: 183-190, 2009.

18. Xiao ZW, Zhang W, Ma L and Qiu ZW: Therapeutic effect of magnesium isoglycyrrhizinate in rats on lung injury induced by paraquat poisoning. Eur Rev Med Pharmacol Sci 18: 311-320, 2014.

19. Chen KJ, Chen WY, Chen X, Jia YM, Peng GQ and Chen L: Increased elimination of paclitaxel by magnesium isoglycyrrhizinate in epithelial ovarian cancer patients treated with paclitaxel plus cisplatin: a pilot clinical study. Eur J Drug Metab Pharmacokinet 39: 25-31, 2014.

20. Abe K, Ikeda T, Wake K, Sato T, Sato T and Inoue H: Glycyrrhizin prevents of lipopolysaccharide/D-galactosamine-induced liver injury through down-regulation of matrix metalloproteinase-9 in mice. J Pharm Pharmacol 60: 91-97, 2008.

21. Ziamajidi N, Khaghani S, Hassanzadeh G, Vardasbi S, Ahmadian S, Nowrouzi A, Ghaffari SM and Abdirad A: Amelioration by chicory seed extract of diabetes- and oleic acid-induced non-alcoholic fatty liver disease (NAFLD)/non-alcoholic steatohepatitis (NASH) via modulation of PPAR a and SREBP-1. Food Chem Toxicol 58: 198-209, 2013.

22. Alkhatatbeh MJ, Lincz LF and Thorne RF: Low simvastatin concentrations reduce oleic acid-induced steatosis in HepG2 cells: An in vitro model of non-alcoholic fatty liver disease. Exp Ther Med 11: 1487-1492, 2016.

23. Malhi H, Bronk SF, Werneburg NW and Gores GJ: Free fatty acids induce JNK-dependent hepatocyte lipoapoptosis. J Biol Chem 281: 12093-12101, 2006.

24. Henkel A and Green RM: The unfolded protein response in fatty liver disease. Semin Liver Dis 33: 321-329, 2013.

25. Marciniak SJ, Yun CY, Oyadomari S, Novoa I, Zhang Y, Jungreis R, Nagata K, Harding HP and Ron D: CHOP induces death by promoting protein synthesis and oxidation in the stressed endoplasmic reticulum. Genes Dev 18: 3066-3077, 2004.

26. Yoshida H, Matsui T, Yamamoto A, Okada T and Mori K: XBP1 mRNA is induced by ATF6 and spliced by IRE1 in response to ER stress to produce a highly active transcription factor. Cell 107: 881-891, 2001. 
27. Adachi Y, Yamamoto K, Okada T, Yoshida H, Harada A and Mori K: ATF6 is a transcription factor specializing in the regulation of quality control proteins in the endoplasmic reticulum. Cell Struct Funct 33: 75-89, 2008.

28. Cao J, Dai DL, Yao L, Yu HH, Ning B, Zhang Q, Chen J, Cheng WH, Shen W and Yang ZX: Saturated fatty acid induction of endoplasmic reticulum stress and apoptosis in human liver cells via the PERK/ATF4/CHOP signaling pathway. Mol Cell Biochem 364: 115-129, 2012.

29. Deng J, Lu PD, Zhang Y, Scheuner D, Kaufman RJ, Sonenberg N, Harding HP and Ron D: Translational repression mediates activation of nuclear factor kappa $\mathrm{B}$ by phosphorylated translation initiation factor 2. Mol Cell Biol 24: 10161-10168, 2004.

30. Hu P, Han Z, Couvillon AD, Kaufman RJ and Exton JH: Autocrine tumor necrosis factor alpha links endoplasmic reticulum stress to the membrane death receptor pathway through IRE1alpha-mediated NF-kappaB activation and down-regulation of TRAF2 expression. Mol Cell Biol 26: 3071-3084, 2006.

31. Urano F, Wang X, Bertolotti A, Zhang Y, Chung P, Harding HP and Ron D: Coupling of stress in the ER to activation of JNK protein kinases by transmembrane protein kinase IRE1. Science 287: 664-666, 2000.
32. Nerstedt A, Cansby E, Amrutkar M, Smith U and Mahlapuu M: Pharmacological activation of AMPK suppresses inflammatory response evoked by IL- 6 signalling in mouse liver and in human hepatocytes. Mol Cell Endocrinol 375: 68-78, 2013.

33. Dooley S and ten Dijke P: TGF- $\beta$ in progression of liver disease. Cell Tissue Res 347: 245-256, 2012.

34. Yang L, Roh YS, Song J, Zhang B, Liu C, Loomba R and Seki E: TGF- $\beta$ signaling in hepatocytes participates in steatohepatitis through regulation of cell death and lipid metabolism. Hepatology 59: 483-495, 2014.

35. Henderson NC and Iredale JP: Liver fibrosis: cellular mechanisms of progression and resolution. Clin Sci (Lond) 112: 265-280, 2007.

36. Marchesini G, Brizi M, Morselli-Labate AM, Bianchi G, Bugianesi E, McCullough AJ, Forlani G and Melchionda N: Association of nonalcoholic fatty liver disease with insulin resistance. Am J Med 107: 450-455, 1999. 